\title{
Posterior Quadratus Lumborum Block versus Subcostal Transversus Abdominis Plane Block in Laparoscopic Cholecystectomy
}

\author{
Hazem El Sayed Moawad Weheba, MD*, Tamer Abdelsalam, MSc, Sameh Ghareeb, \\ MD and Mohamed Younis Makharita, MD
}

Department of Anesthesia and Surgical Intensive Care, Mansoura University, Egypt

*Corresponding author: Hazem El Sayed Moawad Weheba, MD, Assistant Professor, Department of Anesthesia And Surgical Intensive Care, Faculty of Medicine, Mansoura University, Egypt, Tel: +021121516041

\begin{abstract}
Background and objectives: Effective perioperative analgesia with laparoscopic cholecystectomy enhances early recovery, ambulation, and discharge. Subcostal TAP block has been shown to reduce perioperative opioid use and provide effective perioperative analgesia. Currently, the QLB is performed as one of the perioperative pain management procedures for patients undergoing abdominal surgery. In the current study, we hypothesized that the analgesic efficacy of posterior QLB would be equal to or better than the subcostal TAP block in laparoscopic cholecystectomy.
\end{abstract}

Methods: 106 patients were randomized for elective laparoscopic cholecystectomy. They were randomly allocated to 2 equal groups, 53 patients each. First group, patients received posterior QLB. Second group, patients received subcostal TAP block. The study was registered at ClinicalTrials.gov (NCT03323684).

Results: Data from 98 patients were analyzed (48 patients in QLB group and 50 patients in the TAP group). The cumulative postoperative fentanyl consumption at 24 hours in patients required postoperative opioids shows no significant difference between the two groups but with less number of patients needing postoperative opioids in QLB group (17/48) than in the TAP group $(28 / 50)$. The time to the first postoperative request for rescue analgesia was significantly longer in QLB group than in the TAP group. There was no significant difference between the two groups as regard PONV, and pain scores at 1, 6, 12, and 24 hours postoperatively.

Conclusion: Posterior quadratus lumborum block can provide better effective postoperative analgesia in patients undergoing laparoscopic cholecystectomy than subcostal transversus abdominis plane block does.

\section{Introduction}

In laparoscopic cholecystectomy, overall pain is a conglomerate of three different and clinically separate components: Incisional pain (somatic pain) due to trocar insertion sites, visceral pain (deep intra-abdominal pain), and shoulder pain (presumably referred visceral pain) [1]. Without effective treatment, this ongoing pain may delay recovery, mandate inpatient admission, and thereby increase the cost of such care [2]. Moreover, it has been hypothesized that intense acute pain after laparoscopic cholecystectomy may predict development of chronic pain (e.g., postlaparoscopic cholecystectomy syndrome) [3].

The uses of peripheral nerve blocks that deliver local anesthetic into the transversus abdominis fascial plane have become popular for operations that involve incision(s) of the abdominal wall. Thus, the Transversus Abdominis plane (TAP) block has been shown to reduce perioperative opioid use in elective abdominal surgery [4] including laparoscopic cholecystectomy [5]. However, the efficacy of the TAP block is reportedly only reliable in providing analgesia below the umbilicus. The ultrasound-guided subcostal transversus abdominis plane (STAP) block is a recently described variation on the TAP block which produces reliable supraumbilical analgesia. Deposition of local anesthetic in this plane has shown to block the thoracolumbar intercostal nerves which are derived from anterior divisions of spinal segmental nerves T6 to $L 1$ anesthetising somatic nerves of abdominal wall but visceral pain following surgery is still an issue $[6,7]$.

Citation: Weheba HESM, Abdelsalam T, Ghareeb S, Makharita MY (2019) Posterior Quadratus Lumborum Block versus Subcostal Transversus Abdominis Plane Block in Laparoscopic Cholecystectomy. Int J Anesthetic Anesthesiol 6:093. doi.org/10.23937/2377-4630/1410093

Accepted: August 06, 2019: Published: August 08, 2019

Copyright: (C) 2019 Weheba HESM, et al. This is an open-access article distributed under the terms of the Creative Commons Attribution License, which permits unrestricted use, distribution, and reproduction in any medium, provided the original author and source are credited. 
Currently, the quadratus lumborum block (QLB) is performed as one of the perioperative pain management procedures for all generations (pediatrics, pregnant, and adult) undergoing abdominal surgery. The local anesthetic injected via the approach of the posterior QLB ( QLB type-2) can more easily extend beyond the TAP to the thoracic paravertebral space or the thoracolumbar plane [8], the posterior QLB entails a broader sensory-level analgesic and may generate analgesia from T7 to L1 [9]. In this study, the posterior QLB (type 2) was compared with the subcostal TAP block in laparoscopic cholecystectomy.

\section{Methods}

Ethical approval for this study was provided by the Mansoura faculty of medicine Institutional review board, Egypt. The study was registered with a clinical trials registry (ClinicalTrials.gov identifier NCT03323684). The study was conducted at the Gastro-intestinal surgery center (Mansoura University) between October $1^{\text {st }}$ 2017 and March $15^{\text {th }}$ 2018. This study is presented in accordance with the Consolidated Standards of Reporting Trials (CONSORT) guidelines. Inclusion criteria were patients programmed for elective laparoscopic cholecystectomy, ASA I or II, and age over 18 years and less than 60-years-old. Exclusion criteria include patient refusal, bleeding or coagulation abnormality, local skin infection and sepsis at site of the block, known hypersensitivity to the study drugs, body Mass Index $>40 \mathrm{~kg} / \mathrm{m}^{2}$, emergency laparoscopic cholecystectomy, and if laparoscopic cholecystectomy turned into open cholecystectomy.

Eligible 106 patients were randomly allocated by the sealed opaque envelope method according to the anesthetic technique used into 2 equal groups (QLB and TAP groups). All patients were assessed preoperatively by history, physical examination, basal laboratory investigations (complete blood picture, INR, liver functions, and kidney functions tests), ECG, and ECHO if needed. The day before the surgery all patients were familiar with the use of visual analogue scale score identifying 0 as no pain and 10 as worst imaginable pain. Demographic data as age, sex, and BMI were registered.

On arrival to operating room, peripheral intravenous cannula (18-20 G) was inserted and $500 \mathrm{ml}$ ringer solution started to be infused. Standard monitoring started by electrocardiography (ECG), non-invasive blood pressure, and pulse oximetry. General anesthesia was then induced using IV propofol at a dose of $2 \mathrm{mg} . \mathrm{kg}$, fentanyl $1 \mu \mathrm{g} . \mathrm{kg}$, atracurium besylate $0.5 \mathrm{mg} . \mathrm{kg}$ to facilitate endotracheal intubation. Patients were then mechanically ventilated to maintain end tidal $\mathrm{CO}_{2}$ around $35 \mathrm{mmHg}$. Anesthesia was then maintained using isoflurane $1 \%$, and $60 \%$ air in oxygen mixture and top up doses of atracurium. Intravenous fluids were given per body weight and according to intraoperative loss.

After induction of general anesthesia, Paracetamol infusion (15 mg.kg) was given by intravenous infusion to all patients in both groups. In both groups, the block was performed and patients received $20 \mathrm{~mL}$ of $0.375 \%$ isobaric bupivacaine for each side and we waited 15 minutes before surgery start. Fentanyl boluses $(0.5$ $\mu \mathrm{g} . \mathrm{kg}$ ) was given in case of increase in intraoperative mean arterial blood pressure or heart rate of more than $20 \%$ of baseline for longer than 5 minutes and dose given was registered. The operation was maintained with normal pressure $\mathrm{CO}_{2}$ pneumoperitoneum between 10 and $12 \mathrm{mmHg}$ and conventional 4-port laparoscopic cholecystectomy. The four ports for the surgery were placed above the umbilicus, below the xiphoid process. These port sites are located between the T6 and T10 dermatomes. All patients were extubated at the end of surgery after neuromuscular reversal. The duration of the surgery was recorded.

In PACU, Ketorolac $30 \mathrm{mg}$ ampoule was given by intravenous infusion to all patients and then every 8 hours in the first postoperative 24 hours. Sensory assessment to evaluate the success of the blockade was undertaken at $1 \mathrm{~h}$ postoperatively by pinprick method using blunt needle with minimal skin deformation testing presence or absence of sensory changes from T4- L2 when compared to C3-4 dermatomes. Blockade was considered successful when there were sensory changes at T6-7 down to T10-11 dermatomes. Criteria for discharge of patients from PACU are: pain control (VAS $\leq 3$ ), absence of nausea and vomiting, hemodynamic stability, and alert or appropriately responsive to voice and duration of stay at PACU was recorded. Pain was assessed using VAS at $1,6,12,24$ hours postoperatively. Any patient deemed to have nausea and or vomiting was recorded. Duration of analgesia was considered as the time interval from an immediate post-operative period until VAS score reached 4 . Fentanyl boluses $(20 \mu \mathrm{g})$ was given if VAS is more than 3 and it might be repeated after 30 minutes until VAS is $\leq 3$ and total dose of fentanyl given was recorded.

In posterior QLB group (type 2), the patients were put in the lateral position and a linear 6-13 $\mathrm{MHz}$ ultrasound transducer (Toshiba SSA- 660A, Japan) was placed in the anterior axillary line to visualize the typical triple anterior abdominal layers. Then, the probe was placed in the midaxillary line and at this juncture the anterior abdominal layers started to taper. When the probe was placed in the posterior axillary line as per the posterior approach, sonoanatomy showed first the transversus abdominis disappearing then the internal oblique and external oblique forming aponeurosis and appearance of QL noticed. The posterior aspect of the QL muscle was confirmed, and a 22-gauge $100 \mathrm{~mm}$ spinal needle was then guided, in plane, and the needle tip was inserted into this aspect of the QL muscle. Following aspiration, the local anesthetic was then injected into the LIFT (lumbar interfascial triangle) behind the QL muscle. 
The thoracolumbar fascia consists of 3 layers: An anterior, middle, and a posterior lumbar fascia. The anterior lumber fascia is the extension of the transversalis fascia; it passes anterior to the quadratus lumborum. The middle lumbar fascia passes between the paraspinal muscles and the quadratus lumborum. The posterior lumbar fascia has a superficial and deep lamina. In the area where the middle lumbar fascia joins the deep lamina of the posterior layer (paraspinal retinacular sheath) on the lateral border of the erector spinae, a triangular structure named the lumbar interfascial triangle is created and this is our target [8].

In the subcostal TAP group, the patients were put in the supine position and a linear $6-13 \mathrm{MHz}$ ultrasound transducer (Toshiba SSA- 660A, Japan) was placed in the midline of the abdomen $2 \mathrm{~cm}$ below the xiphisternum and moved laterally along the subcostal margin. The transversus abdominis muscle was identified lying beneath and extending lateral to the rectus abdominis muscle. A 22-gauge $100 \mathrm{~mm}$ spinal needle is introduced in plane with the probe positioned perpendicular to the abdominal wall, directed parallel to the costal margin but oblique to the sagittal plane. The needle insertion point is near the xiphoid process and the local anesthetic is initially deposited between transversus abdominis and the rectus abdominis muscles. The needle is then directed cautiously inferolaterally to progressively distend the transversus abdominis plane parallel to the costal margin blocking the intercostal nerves as they emerge to run into the transversus plane from the xiphoid process towards the anterior part of the iliac crest [10].

Using a pervious study in TAP block group [11]; the mean \pm SD of cumulative morphine consumption at 24 hours was $16 \pm 9.9 \mathrm{mg}$. Assuming $\alpha=0.05$ and $\beta=$ 0.1 (90\% power) and using the 2-tailed Student t test, 49 subjects will be required in each group to detect a $4 \mathrm{mg}$ reduction in the mean cumulative morphine consumption, which will be considered the minimal clinically significant effect. To allow for dropouts, 53 subjects will be assigned to each group with a total sample size of 106 patients.

For statistical analysis SPSS version 22 was used.

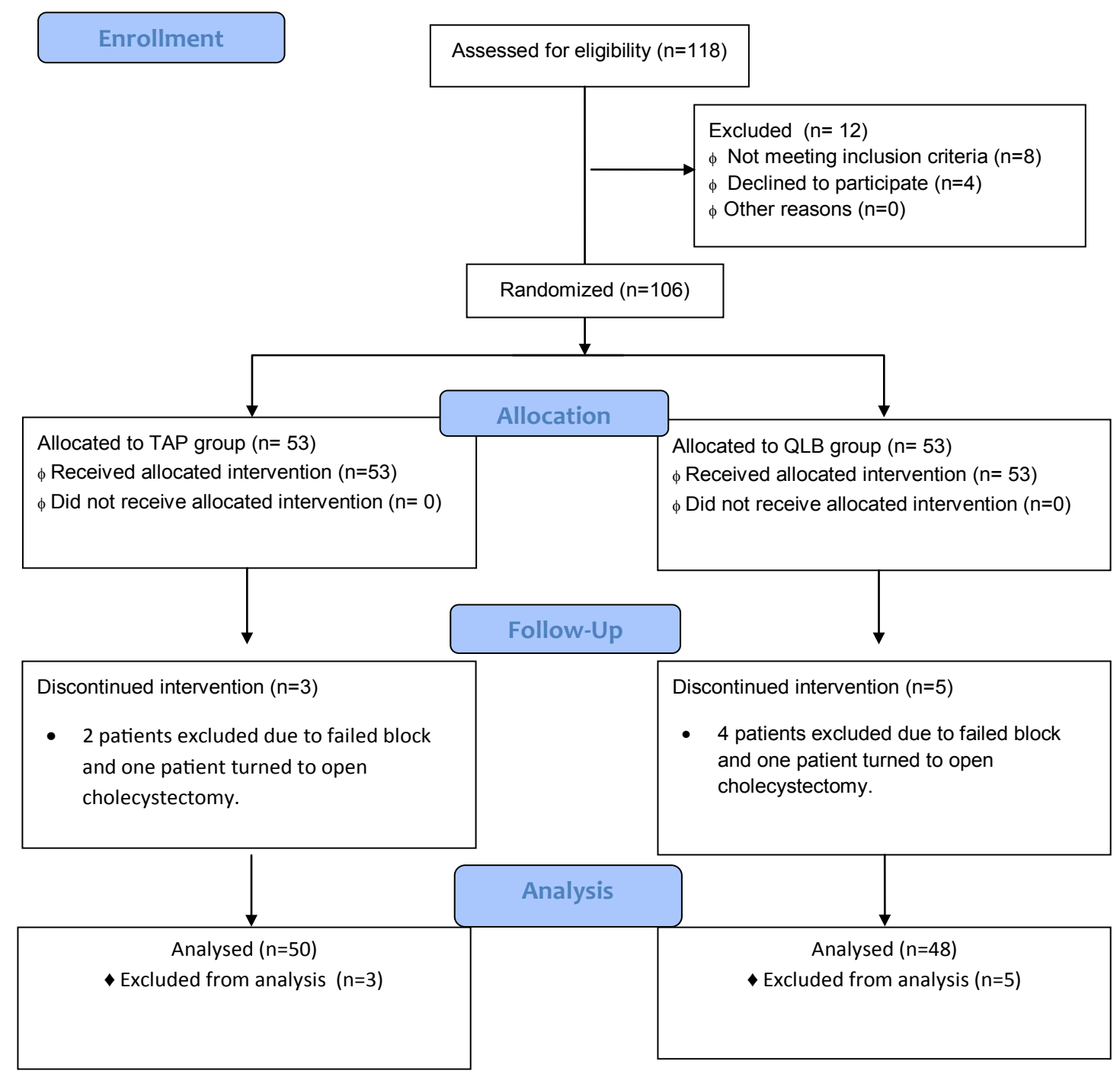

Figure 1: CONSORT 2010 flow diagram. 
Differences between the two groups were analyzed by student's t-test for normally distributed continous data and were presented as mean \pm standard deviation and the Mann-Whitney $U$ test for data without normal distribution and were presented as median (range). For categorical data, the chi-square test was used and was presented as number (percentage). P-value $<0.05$ was considered statistically significant.

\section{Results}

106 patients were randomized, and data from 98 patients (48 patients in QLB group and 50 patients in the TAP group) were analyzed (Figure 1). Five patients were excluded from the analysis in QLB group, and three patients were excluded from the analysis in the TAP group.

There were no clinically significant differences between the 2 groups as regard age, sex, BMI, and duration of surgery (Table 1 ).

There was no significant difference in VAS at 1, 6, 12, 24 hours postoperatively (Table 2).

There was no significant difference in cumulative fentanyl consumption at 24 hours between the two groups $(P=0.527)$. The postoperative cumulative fentanyl consumption at 24 hours in patients required postoperative opioids was $58.82 \pm 11.11 \mu \mathrm{g}$ in the QLB group and $62.14 \pm 17.50 \mu \mathrm{g}$ in the TAP group (Table 3).

There was no significant difference between the two groups as regard intraoperative fentanyl consumption $(40 \pm 7.07 \mu \mathrm{g}$ in the QLB group and $42 \pm 10.36 \mu \mathrm{g}$ in the TAP group, $P=0.802$ ). The time to the first postoperative request for rescue analgesia was significantly longer in QLB group (420.58 \pm 114.76 minutes) than in the TAP group (267.32 \pm 62.32 minutes) $(P=0.000)$ (Table 3$)$.

31 out of 48 patients (64.58\%) did not require postoperative fentanyl in the QLB group and 22 out of 50 patients (44\%) did not require postoperative fentanyl in the TAP group ( $P=0.03$ ). Also, there was no significant difference between the two groups as regard length of stay in PACU (38.33 \pm 7.60 minutes in QLB group and $39.60 \pm 8.38$ minutes in the TAP group, $P=0.505)$. There was no significant difference between the two groups as regard the incidence of PONV (P=0.375) (Table 4).

\section{Discussion}

To the best of our knowledge, this is one of the first randomized controlled trials comparing posterior QLB versus subcostal TAP block for postoperative pain relief after laparoscopic cholecystectomy. As a part of multimodal analgesia, the results of the current study showed that the posterior QLB was a superior analgesic technique compared to subcostal TAP block for post-

Table 1: Demographic data (age, sex, BMI, and duration of surgery) in the studied groups.

\begin{tabular}{|l|l|l|l|}
\hline & QLB group $(\mathbf{n}=\mathbf{4 8})$ & TAP group $(\mathbf{n}=\mathbf{5 0})$ & P value \\
\hline Age $($ years) Mean \pm SD & $41.97 \pm 11.55$ & $41.70 \pm 11.56$ & 0.955 \\
\hline Sex $(\mathrm{F} / \mathrm{M})$ & $36 / 12$ & $33 / 17$ & 0.276 \\
\hline BMI $\left(\mathrm{Kg} / \mathrm{m}^{2}\right)$ Mean \pm SD & $30.68 \pm 3.06$ & $31.50 \pm 4.18$ & 0.281 \\
\hline Surgery duration $(\mathrm{min})$ Mean \pm SD & $36.66 \pm 8.40$ & $36.80 \pm 6.52$ & 0.937 \\
\hline
\end{tabular}

Data are expressed as mean \pm SD, number. P-value < 0.05 was considered statistically significant. QLB: Quadratuslumborum block group, TAP: Transversus abdominis plane block group, BMI: body mass index, F: Female, M: Male.

Table 2: Postoperativevisual analogue scale (VAS) score at 1,6, 12, and 24 hours in the studied groups.

\begin{tabular}{|l|l|l|l|}
\hline & $\begin{array}{l}\text { QLB group } \\
(\mathbf{n = 4 8 )}\end{array}$ & $\begin{array}{l}\text { TAP group } \\
(\mathbf{n = 5 0 )}\end{array}$ & 0.323 \\
\hline $1 \mathrm{~h}$ & $1.00(0-3)$ & $1.00(0-3)$ & 0.128 \\
\hline $6 \mathrm{~h}$ & $1.00(0-5)$ & $2.00(0-6)$ & 0.722 \\
\hline $12 \mathrm{~h}$ & $1.00(0-5)$ & $1.00(0-4)$ & 0.244 \\
\hline $24 \mathrm{~h}$ & $1.00(0-2)$ & $1.00(0-2)$ & \\
\hline
\end{tabular}

Data are expressed as median (range). P-value < 0.05 was considered statistically significant. QLB: Quadratus lumborum block group, TAP: Transversus abdominis plane block group.

Table 3: Intraoperative and postoperative fentanyl consumption, length of stay in PACU, and time of first request to rescue analgesia in both groups.

\begin{tabular}{|l|l|l|l|}
\hline & QLB $(\mathbf{n}=\mathbf{4 8})$ & TAP $(\mathbf{n}=\mathbf{5 0})$ & P value \\
\hline Intraoperative fentanyl consumption $(\mu \mathrm{g})$ & $40 \pm 7.07$ & $42 \pm 10.36$ & 0.802 \\
\hline Cumulative postoperative fentanyl consumption $(24 \mathrm{~h})(\mu \mathrm{g})$ & $58.82 \pm 11.11$ & $62.14 \pm 17.50$ & 0.527 \\
\hline Time of first request to rescue analgesia (minutes) & $420.58 \pm 114.76$ & $267.32 \pm 62.32$ & 0.000 \\
\hline
\end{tabular}

Data are expressed as mean $\pm \mathrm{SD}$, median (range). P-value $<0.05$ was considered statistically significant. QLB: Quadratus lumborum block group, TAP: Transversus abdominis plane block group. 
Table 4: Number and percentage of patients who did not require postoperative fentanyl, length of stay in PACU, and PONV (number and perentage) in both groups.

\begin{tabular}{|c|c|c|c|}
\hline & $\begin{array}{l}\text { QLB } \\
(n=48)\end{array}$ & $\begin{array}{l}\text { TAP } \\
(n=50)\end{array}$ & P value \\
\hline Number and percentage of patients did not require postoperative fentanyl & $31(64.58 \%)$ & $22(44 \%)$ & 0.03 \\
\hline length of stay in PACU (minutes) & $38.33 \pm 7.60$ & $39.60 \pm 8.38$ & 0.505 \\
\hline PONV (number and perentage) & $18(37.5 \%)$ & $18(36 \%)$ & 0.375 \\
\hline
\end{tabular}

Data are expressed as mean $\pm S D$, number (percentage). P-value $<0.05$ was considered statistically significant. QLB: Quadratus lumborum block group, TAP: Transversus abdominis plane block group.

operative pain relief after laparoscopic cholecystectomy, with significant higher number of patients who did not require postoperative opioids and delaying the time of the first request to rescue analgesia in QLB group.

The primary outcome of this study was to compare the cumulative postoperative opioid requirements at 24 hours. The secondary outcomes were comparing the intraoperative opioid requirements, postoperative visual analogue scale (VAS), length of stay at postanesthesia care unit (PACU), time of first request to rescue analgesia, and incidence of PONV.

There was no significant difference in cumulative fentanyl consumption at 24 hours between the two groups in patients requiring postoperative opioid. 31 out of 48 patients $(64.58 \%$ ) did not require postoperative fentanyl in the QLB group and 22 out of 50 patients (44\%) did not require postoperative fentanyl in the TAP group. Time to the first request for postoperative rescue analgesia was significantly longer in QLB group than in the TAP group. Also, there was no significant difference between the two groups as regard PONV, VAS at 1 , $6,12,24$ hours postoperatively, length of stay in PACU, and intraoperative fentanyl consumption.

Comparative studies have shown that the QLB covers a topographically broader field (T7-L1, compared to TAP T10-T12) [8,9], and yields prolonged pain-free condition compared to the TAP block (24-48 $\mathrm{h}$ QLB versus 8-12 h TAP block) $[8,9,12]$.

Several studies have shown that subcostal TAP block can produce effective perioperative analgesia for laparoscopic cholecystectomy [13-16].

Blanco, et al. [8] in a previous study has compared posterior quadratus lumborum block versus classical transversus abdominis plane block for postoperative pain relief after cesarean delivery. Patients who received QLB had significantly less cumulative morphine doses than patients who received the TAP block $(\mathrm{P}<$ 0.005 ) at 12 hours, 24 hours, and up to 48 hours.

Ökmen, et al. [17] has compared posterior QLB plus IV PCA (as a part of multimodal analgesia) versus IV PCA for postoperative pain after laparoscopic cholecystectomy. The VAS scores between the two groups and the mean values of the quantity of tramadol use at the $6^{\text {th }}$, $12^{\text {th }}$, and $24^{\text {th }}$ hours were found to be statistically significantly lower in QLB plus IV PCA group.
While QLB have been proposed to accomplish somatic as well as visceral analgesia of the abdomen, TAP blockade is limited to somatic anesthesia of the abdominal wall only, so we recommended QLB as an add-on block to reduce the requirement of general anesthetic intraoperatively or it could be used as the main component of multimodal analgesia postoperatively.

The advantages of using a posterior approach for the QLB when compared to the lateral or the anterior approach is a more superficial point of injection, better ultrasonographic resolution and a potentially safer injection [8].

There is currently no general consensus on the mechanism(s) of action of QL blockade. We believe that the thoracolumbar fascia and the spread into the paravertebral space play the primary role in the mechanism of action of the quadrates lumborum block.

TLF is a connective tissue tubular structure enveloping the back muscles, connects the anterolateral abdominal wall with the lumbar paravertebral region. The TLF is on its medial side attached to the thoracic and lumbar vertebrae, cranially continuing with endothoracic, and caudally with the fascia iliaca, potentially ensuring the spread of local anesthetics in the craniocaudal direction [18]. It is believed that the local anesthetics spread along the TLF and the endothoracic fascia into the paravertebral space, is responsible in part for the analgesia. In 2011, Carney, et al. [19] showed that contrast spreads from the L1-T5segment of the paravertebral space. Hence the assumption that visceral analgesia results from the spread of anesthetics to the celiac ganglion or sympathetic trunk via splanchnic nerves, as is the case with the paravertebral block. This remains to be confirmed or denied by future research.

An additional mechanism of action of local anesthetics can be explained by the anatomical-histological characteristics of the TLF. Namely, in the superficial layer of the TLF, there is at thick network of sympathetic neurons. In the fascia, there are the high-threshold and low-threshold mechanoreceptors and pain receptors sensitive to the effects of the local anesthetics. These receptors play a role in the development of both acute and chronic pain. The QLB analgesia could be, at least partially, explained by local anesthetic blockade of these receptors $[20,21]$. 
So far, studies done on cadavers [22-26] show that the injected contrast can spread cranially to the thoracic paravertebral space and intercostal spaces covering somatic nerves and the thoracic sympathetic trunk up to the T4 level. Blockade of the subcostal, iliohypogastric, and ilioinguinal nerve is consistent. Caudally, contrast can reach lumbar nerve roots, but the results vary and new studies are needed to clarify the link between the type of QLB and the achieved analgesic effect.

All of these data indicate that the QLB provides somatic and visceral analgesia. Obviously, there are variations in the width of achieved analgesia, and in the number of dermatomes covered by QLB. In most of the cases, analgesia is achieved in T7-L1 dermatomes [8,2630], although there are descriptions of cranial spread to T4-T5 [31], and caudal spread to L2-L3 [24] dermatomes.

This study limitations include the need for an assistant for the lateral positioning of the patient for QLB after general anesthesia and we recommend longer duration of follow-up in future research to assess the effect on chronic pain management. Also we recommend evaluation of stress response indicators like serum cortisol, sugar in future studies.

\section{References}

1. Bisgaard T, Klarskov B, Rosenberg J, Kehlet H (2001) Characteristics and prediction of early pain after laparoscopic cholecystectomy. Pain 90: 261-269.

2. Alexander JI (1997) Pain after laparoscopy. Br J Anaesth 79: 369-378.

3. Bisgaard T, Rosenberg J, Kehlet H (2005) From acute to chronic pain after laparoscopic cholecystectomy: A prospective follow-up analysis. Scand J Gastroenterol 40: 1358-1364.

4. Kadam RV, Field JB (2011) Ultrasound-guided continuous transverse abdominis plane block for abdominal surgery. $\mathrm{J}$ Anaesthesiol Clin Pharmacol 27: 333-336.

5. El-Dawlatly AA, Turkistani A, Kettner SC, Machata AM, Delvi MB, et al. (2009) Ultrasound guided transversus abdominis plane block: Description of a new technique and comparison with conventional systemic analgesia during laparoscopic cholecystectomy. Br J Anaesth 102: 763-767.

6. Hebbard PD, Barrington MJ, Vasey C (2010) Ultrasoundguided continuous oblique subcostal transversus abdominis plane blockade: Description of anatomy and clinical technique. Reg Anesth Pain Med 35: 436-441.

7. Hebbard P, Fujiwara Y, Shibata Y, Royse C (2007) Ultrasound-guided transversus abdominis plane (TAP) block. Anaesth Intensive Care 35: 616-617.

8. Blanco R, Ansari T, Riad W, Shetty N (2016) Quadratus lumborum block versus transversus abdominis plane block for postoperative pain after cesarean delivery: A randomized controlled trial. Reg Anesth Pain Med 41: 757-762.

9. Murouchi T, Iwasaki S, Yamakage M (2016) Quadratus lumborum block: Analgesic effects and chronological ropivacaine concentrations after laparoscopic surgery. Reg Anesth Pain Med 41: 146-150.

10. Hebbard $P$ (2008) Subcostal transversus abdominal plane block under ultrasound guidance. Anesth Analg 106: 674675.
11. Ortiz J, Suliburk JW, Wu K, Bailard NS, Mason C, et al. (2012) Bilateral transversus abdominis plane block does not decrease postoperative pain after laparoscopic cholecystectomy when compared with local anesthetic infiltration of trocar insertion sites. Reg Anesth Pain Med 37: 188-192.

12. Oksuz G, Bilal B, Gurkan Y, Urfalioğlu A, Arslan M, et al. (2017) Quadratus lumborum block versus transversus abdominis plane block in children undergoing low abdominal surgery: A randomized controlled trial. Reg Anesth Pain Med 42: 674-679.

13. Tolchard S, Davies R, Martindale S (2012) Efficacy of the subcostal transversus abdominis plane block in laparoscopic cholecystectomy: comparison with conventional port-site infiltration. J Anaesthesiol Clin Pharmacol 28: 339-343.

14. Bhatia N, Arora S, Jyotsna W (2014) Comparison of posterior and subcostal approaches to ultrasound-guided transverse abdominis plane block for postoperative analgesia in laparoscopic cholecystectomy. J Clin Anesth 26: 294-299.

15. Chen CK, Tan PC, Phui VE, Shu Ching T (2013) A comparison of analgesic efficacy between oblique subcostal transversus abdominis plane block and intravenous morphine for laparascopic cholecystectomy. A prospective randomized controlled trial. Korean J Anesthesiol 64: 511-516.

16. Shin HJ, Oh AY, Baik JS, Kim JH, Han SH, et al. (2014) Ultrasound-guided oblique subcostal transversus abdominis plane block for analgesia after laparoscopic cholecystectomy: A randomized, controlled, observerblinded study. Minerva Anestesiol 80: 185-193.

17. Ökmen K, Metin Ökmen B, Topal S (2018) Ultrasoundguided posterior quadratus lumborum block for postoperative pain after laparoscopic cholecystectomy: A randomized controlled double blind study. J Clin Anesth 49: 112-117.

18. El-Boghdadly K, Elsharkawy H, Short A, Chin KJ (2016) Quadratus lumborum block nomenclature and anatomical considerations. Reg Anesth Pain Med 41: 548-549.

19. Carney J, Finnerty O, Rauf J, Bergin D, Laffey JG, et al. (2011) Studies on the spread of local anaesthetic solution in transversus abdominis plane blocks. Anaesthesia 66: 1023-1030.

20. Benetazzo L, Bizzego A, De Caro R, Frigo G, Guidolin D, et al. (2011) 3D reconstruction of the crural and thoracolumbar fasciae. Surg Radiol Anat 33: 855-862.

21. Tesarz J, Hoheisel U, Wiedenhofer B, Mense S (2011) Sensory innervation of the thoracolumbar fascia in rats and humans. Neuroscience 194: 302-308.

22. Kumar A, Sadeghi N, Wahal C, Gadsden J, Grant SA (2017) Quadratus lumborum spares paravertebral space in fresh cadaver injection. Anesth Analg 125: 708-709.

23. Dam M, Moriggl B, Hansen CK, Hoermann R, Bendtsen TF, et al. (2017) The pathway of injectate spread with the transmuscular quadratus lumborum block: A cadaver study. Anesth Analg 125: 303-312.

24. Carline L, McLeod GA, Lamb C (2016) A cadaver study comparing spread of dye and nerve involvement after three different quadratus lumborum blocks. $\mathrm{Br} \mathrm{J}$ Anaesth 117: 387-394.

25. Adhikary SD, El-Boghdadly K, Nasralah Z, Sarwani N, Nixon AM, et al. (2017) A radiologic and anatomic assessment of injectate spread following transmuscular quadratus lumborum block in cadavers. Anaesthesia 72: 73-79. 
26. Elsharkawy H, El-Boghdadly K, Kolli S, Esa WAS, DeGrande S, et al. (2017) Injectate spread following anterior subcostal and posterior approaches to the quadratus lumborum block: A comparative cadaveric study. Eur J Anaesthesiol 34: 587-595.

27. Borglum J, Moriggl B, Jensen K, Lonnqvist PA, Christensen $A F$, et al. (2013) Ultrasound-guided transmuscular quadratus lumborum blockade. $\mathrm{Br} \mathrm{J}$ Anaesth 111.

28. Ueshima H, Hiroshi O (2017) Intermittent bilateral anterior subcostal quadratus lumborum block for effective analgesia in lower abdominal surgery. J Clin Anesth 43: 65.
29. Ueshima H, Otake H, Lin JA (2017) Ultrasound-guided quadratus lumborum block: An updated review of anatomy and techniques. Biomed Res Int.

30. Kadam VR (2013) Ultrasound-guided quadratus lumborum block as a postoperative analgesic technique for laparotomy. J Anaesthesiol Clin Pharmacol 29: 550-552.

31. Chin KJ, McDonnell JG, Carvalho B, Sharkey A, Pawa $A$, et al. (2017) Essentials of our current understanding: abdominal wall blocks. Reg Anesth Pain Med 42: 133-183. 